\title{
Article
}

\section{Removal of Trace Level Metal Impurities from Ultrapure Water by Filter Cartridge Including Ion Exchange Grafted Membrane}

\author{
Masanori KANAZAWA \\ Research and Development, Lab Water Division, Nihon Millipore K.K., 1-4-28 Mita, Minato-ku, Tokyo 108-0073, \\ Japan
}

Yukio HASHIMOTO

Application Development, Nihon Mykrolis K.K., 1-4-28 Mita, Minato-ku, Tokyo 108-0073, Japan

(Manuscript submitted February 4, 2003; accepted April 10, 2003)

\begin{abstract}
Metal removal performance of filter cartridge with bi-layered structure was investigated. The filter was prepared using UHMWPE and ion exchange membrane made by grafting polymerization. In filtration experiment, feed ultrapure water was controlled spiking of 15 metals at ppt level, and removal performance of each metal was determined using ICP-MS measurement of filtrate. Metal adsorption behavior for most of the metals were following the classical fluid dynamic theory and the boundary layer controlled reaction. The results were compared to conventional UHMWPE membrane filter and discussed for each metal.
\end{abstract}

\section{Introduction}

Ultrapure water (UPW) is the most commonly used chemical species in many manufacturing industries including microelectronics fabrication. Regarding the use of water, there are two technical difficulties that need to be overcome to improve their production qualities. The first one is the sensitivity of monitoring systems. Resistivity monitor is the most commonly monitoring sensor used to detect the presence of inorganics. However, simple and low cost sensor is not enough sensitivity level for UPW used for microelectronics. For example, in case the resistivity falls down from $18.2 \mathrm{M} \Omega \mathrm{cm}$ to $18.0 \mathrm{M} \Omega \mathrm{cm}$, impurity contents increase by $300 \mathrm{ng} / \mathrm{dm}^{3}$ (ppt) as $\mathrm{NaCl}$ equivalent. [1] This $300 \mathrm{ng} / \mathrm{dm}^{3}$ of impurity in UPW is above the alert level in microelectronics device fabrication process. The second issue is that in most manufacturing plant the water quality is not as good as at the point of use as it is right after the UPW purification system. UPW at the point of use has a considerable level of contamination due to exposure to long pipeline and manufacturing tools. Even if the quality of UPW right after the UPW system meets the specification, UPW gets contaminated before it touches silicon wafer. In order to solve these problems, a Point of Use (POU) filter cartridge was developed to purify UPW before it touches the wafer. In this report, the metal removal performance was investigated and compared for two different filter cartridges. The first filter cartridge contained a bi-layered structure of two different membranes. One is an ultrahigh molecular weight polyethylene (UHMWPE) membrane with $0.1 \mu \mathrm{m}$ micropore and the second is a cation exchange membrane prepared by grafting polymerization. The second filter cartridge was made from conventional UHMWPE membrane only. The metal removal performances were compared for different metal concentrations and the behavior of both filters was discussed. 


\section{Experimental}

\subsection{Sample Cartridges}

The sample filter cartridge was assembled as pleated structure with two layers of membranes, the first layer was a cation exchange membrane with styrene sulfonic acid group as a functional group and the second layer was an ultra-high-molecular-weight-polyethylene (UPMWPE) membrane. The cation exchange membrane was prepared by introducing functional group onto the polyethylene as the base material. The grafting polymerization process is summarized as follow; the base membrane is irradiated with an electron beam, and activated radicals were created on the surface. The membrane was exposed to the monomer solution containing the functional group on the side chain. The sulfonic group become the ion exchange group. Styrene sulfonic acid is the most favorable functional group as a strong cation exchange function. [2] The ion exchange capacity is $0.02 \mathrm{meq} / \mathrm{cm}^{2}$. The second layer was a $0.1 \mu \mathrm{m}$ pore size membrane which surface was modified by hydrophilic weak acid groups. The membrane surface area was $1,200 \mathrm{~cm}^{2}$ and the housing material was high-density-polyethylene (HDPE)(following ion exchange cartridge). In this study, the single layer $0.1 \mu \mathrm{m}$ hydrophilic UHMWPE filter cartridge had a surface area of $1,800 \mathrm{~cm}^{2}$ (following UHMWPE cartridge) and was used for same experiment.

\subsection{Filtration Experimental System}

Figure 1 is a configuration of filtration experiment system. Feed water was municipal water in Mita, Minato-ku, Tokyo. It was purified by reverse osmosis (RO) followed by electro deionization (EDI) to above $15 \mathrm{M}$ $\Omega \mathrm{cm}$ in pure water system (Millipore, Elix-10), and was stored in the polyethylene tank. The pure water was further purified by two identical ultrapure water system (Millipore, Milli-Q Element) installed in parallel. The ultrapure water system contains a circulation pump, an ultraviolet lamp and two sets of cartridge polisher, the effluent UPW achieved $18.2 \mathrm{M} \Omega \mathrm{cm}$ in resistivity and less than $5 \mathrm{ppb}$ of total organic carbon (TOC). Two sample filter cartridges were placed close to point of use on the UPW line for the experiment. The diluted multi-element standard solution (SPEX, XSTC-331 Lot 12-05PD, $100 \mu \mathrm{g} / \mathrm{dm}^{3}$ ), was injected through tubing between UPW system and sample cartridge to feed metal impurity with controlled concentration. Flow rate of the ultrapure water was $1.2 \mathrm{dm}^{3} / \mathrm{min}$.

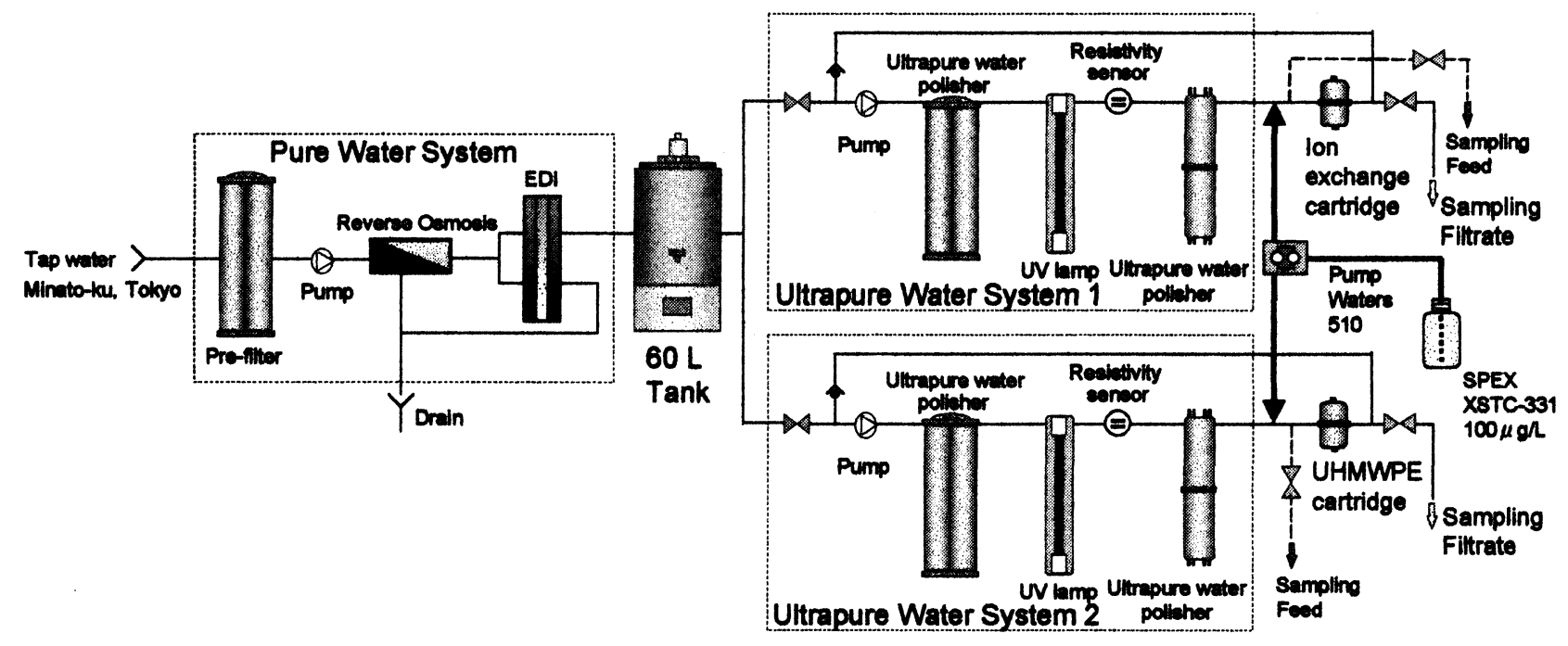

Fig. 1 Configuration of filtration experiment system

\subsection{Start up Property and Cleanliness}

In order to reach maximum performances, the preconditioning procedure of the ion exchange cartridge was 
conducted. It is common for ion exchange resin, because the ion exchange membrane needs to be swollen with the fluid used for filtration before use. The details of the installation procedure were as follows. The UPW was filled to the dry cartridge sample and flushed for $5 \mathrm{~min}$, and sample cartridges were allowed to equilibrate by an over-night soaking. In order to confirm the initial cleanliness of the system, UPW was sampled out after 1, 2, 3, 5, 10 and 15 min after the operation started and metal concentration was analyzed by ICP-MS.

\subsection{Metal Removal Experiment}

5 minutes after the UPW system was started, the injection pump was turned on to spike the metal ions. The concentration of each spiked metal was controlled to $20 \mathrm{ng} / \mathrm{dm}^{3}, 50 \mathrm{ng} / \mathrm{dm}^{3}, 100 \mathrm{ng} / \mathrm{dm}^{3}$ and $200 \mathrm{ng} / \mathrm{dm}^{3}$. The filtrate water was sampled out after $3 \mathrm{~min}$ when the concentration had changed. Feed metal spiked solutions were also sampled out and compared. The metal concentration was measured by HP-4500 ICP-MS on $700 \mathrm{~W}$ of $\mathrm{RF}$ power with a signal accumulation time of $1 \mathrm{~s}$ for each element.

\section{Results and Discussion}

\subsection{Cleanliness of the Filter Cartridges}

The metal impurity level in the filtrate of both ion exchange cartridge and UHMWPE cartridge were almost at the same level from $1 \mathrm{~min}$ to $15 \mathrm{~min}$. All elements were $1 \mathrm{ng} / \mathrm{dm}^{3}$ or less and were the minimum concentrations that could be detected by ICP-MS.

\subsection{Metal Removal Test}

The table summarizes each metal concentration of feed and filtrate, and Fig. 2 and 3 show the percent retention (100 $\left.\times\left(C_{\text {feed }}-C_{\text {filtrate }}\right) / C_{\text {feed }}\right)$ of the cartridges. The metal retention of ion exchange cartridge with $20 \mathrm{ng} /$ $\mathrm{dm}^{3}$ in feed was $76-99 \%$, and $97-99 \%$ for $200 \mathrm{ng} / \mathrm{dm}^{3}$ on each element except $\mathrm{Zn}$. For alkaline metal ( $\mathrm{Li}, \mathrm{Na}$ and $\mathrm{K}$ ) and a univalent metal ( $\mathrm{Ag})$, the concentration of filtrate increased simultaneously in similar proportion with the increase of the feed metal concentration. It resulted that the metal retention was maintained at a similar value for different feed metal concentration. For example, with $\mathrm{Li}$ or $\mathrm{Na}$, around $1 \mathrm{ng} / \mathrm{dm}^{3}$ of metal flows through the ion exchange cartridge when the feed was at $20 \mathrm{ng} / \mathrm{dm}^{3}$, and $7 \mathrm{ng} / \mathrm{dm}^{3}$ of metal flows out with the $200 \mathrm{ng} / \mathrm{dm}^{3}$ feed concentration. The concentration of alkaline metals in the filtrate increased simultaneously and proportionally to concentration of alkaline metals in the feed. For alkaline earth metal $(\mathrm{Mg}, \mathrm{Ca})$ and some bivalent and trivalent metals ( $\mathrm{Al}, \mathrm{Cr}, \mathrm{Mn}, \mathrm{Co}, \mathrm{Ni}, \mathrm{Cu}$ and $\mathrm{Pb}$ ), filtrate reduced to minimum detecting limit of ICP-MS, the metal retention were maintained around 97 to $99 \%$ in each feed concentration. It was two to three points higher than alkaline metals, which is the result of the different affinity between ion exchange group and metal ions. Alkaline

Table 1 Performance of ion exchange and UHMWPE cartridges for of metal removal

\begin{tabular}{|c|c|c|c|c|c|c|c|c|c|c|c|c|c|c|c|c|c|}
\hline \multirow{2}{*}{\multicolumn{2}{|c|}{ Inlet }} & \multicolumn{8}{|c|}{ Ion exchange cartridge } & \multicolumn{8}{|c|}{ UHMWPE cartridge } \\
\hline & & \multicolumn{2}{|c|}{$20 \mathrm{ng} / \mathrm{L}$} & \multirow{2}{*}{\multicolumn{2}{|c|}{$50 \mathrm{ng} / \mathrm{L}$}} & \multicolumn{2}{|c|}{$100 \mathrm{ng} / \mathrm{L}$} & \multicolumn{2}{|c|}{$200 \mathrm{ng} / \mathrm{L}$} & \multirow{2}{*}{\multicolumn{2}{|c|}{$20 \mathrm{ngl}$}} & \multirow{2}{*}{\multicolumn{2}{|c|}{$50 \mathrm{ng} / \mathrm{L}$}} & \multicolumn{2}{|c|}{$100 \mathrm{ng} / \mathrm{L}$} & \multicolumn{2}{|c|}{$200 \mathrm{ng} / \mathrm{L}$} \\
\hline mass & element & Filtrate & Feed & & & Filtrate & Feed & Filtrate & Feed & & & & & Filtrate & Feed & Filltrate & Feed \\
\hline 7 & $\mathrm{Li}$ & 0.59 & 22.40 & 1.66 & 56.08 & 3.38 & 110.68 & 7.01 & 216.15 & 22.03 & 21.10 & 53.29 & 52.84 & 107.26 & 104.82 & 210.32 & 210.54 \\
\hline 23 & $\mathrm{Na}$ & 1.12 & 23.30 & 2.26 & 50.44 & 3.58 & 97.20 & 7.17 & 307.05 & 19.32 & 18.65 & 47.34 & 58.25 & 108.86 & 93.40 & 185.83 & 186.00 \\
\hline 24 & $\mathrm{Mg}$ & 0.06 & 21.55 & 0.14 & 23.44 & 0.07 & 89.01 & 0.34 & 211.06 & 1.84 & 24.71 & 8.16 & 54.53 & 61.60 & 63.99 & 231.42 & 220.24 \\
\hline 27 & Al & 0.29 & 4.08 & 0.29 & 12.34 & 0.31 & 36.29 & 0.42 & 57.83 & 0.05 & 15.13 & 0.08 & 48.98 & 0.14 & 30.49 & 0.29 & 100.44 \\
\hline 39 & $\mathrm{~K}$ & 2.23 & 23.41 & 2.37 & 55.28 & 3.67 & 106.95 & 6.57 & 258.90 & 21.88 & 21.35 & 53.35 & 53.45 & 108.99 & 101.99 & 201.61 & 201.82 \\
\hline 40 & $\mathrm{Ca}$ & 7.29 & 31.10 & 2.76 & 27.66 & 3.19 & 97.26 & 3.36 & 233.77 & 3.68 & 34.32 & 9.00 & 62.23 & 65.74 & 59.08 & 278.77 & 220.66 \\
\hline 52 & $\mathrm{Cr}$ & 0.39 & 8.68 & 0.29 & 23.49 & 0.29 & 46.81 & 0.41 & 64.19 & 0.27 & 18.37 & 0.29 & 65.04 & 0.68 & 45.96 & 2.13 & 156.20 \\
\hline 55 & $\overline{M n}$ & 0.25 & 22.39 & 0.24 & 24.34 & 0.29 & 90.75 & 0.41 & 148.10 & 1.49 & 24.11 & 5.80 & 53.25 & 49.07 & 62.36 & 235.77 & 222.94 \\
\hline 56 & $F_{\theta}$ & 0.59 & 4.40 & 0.69 & 10.34 & 0.59 & 31.94 & 0.87 & 76.24 & 0.58 & 4.40 & 1.41 & 10.34 & 5.37 & 31.94 & 12.98 & 97.85 \\
\hline 59 & Co & 0.18 & 21.69 & 0.05 & 25.20 & 0.09 & 87.56 & 0.33 & 148.26 & 1.04 & 24.46 & 5.40 & 54.09 & 47.22 & 63.63 & 234.65 & 224.22 \\
\hline 60 & $\mathrm{Ni}$ & 0.22 & 24.75 & 0.22 & 23.70 & 0.15 & 89.07 & 0.22 & 149.07 & 1.12 & 25.79 & 4.47 & 54.34 & 40.92 & 63.35 & 231.43 & 221.15 \\
\hline 63 & $\mathrm{Cu}$ & 0.06 & 7.32 & 0.03 & 17.74 & 0.06 & 69.28 & 0.22 & 140.16 & 0.06 & 27.07 & 0.06 & 52.66 & 0.99 & 53.62 & 4.86 & 224.02 \\
\hline 67 & $\mathrm{Zn}$ & 18.52 & 19.04 & 33.45 & 51.97 & 41.68 & 112.69 & 67.41 & 106.00 & 15.95 & 20.58 & 45.80 & 49.91 & 95.71 & 103.43 & 202.75 & 208.92 \\
\hline 107 & $\overline{A q}$ & 0.15 & 21.80 & 1.02 & 56.50 & 2.20 & 114.14 & 5.83 & 208.57 & 1.02 & 21.70 & 26.41 & 53.95 & 96.89 & 106.66 & 198.95 & 213.18 \\
\hline 208 & $\mathrm{~Pb}$ & 0.19 & 6.58 & 0.37 & 16.40 & 0.28 & 50.03 & 0.09 & 118.22 & 0.09 & 46.05 & 0.19 & 55.78 & 0.19 & 41.69 & 0.46 & 248.40 \\
\hline
\end{tabular}




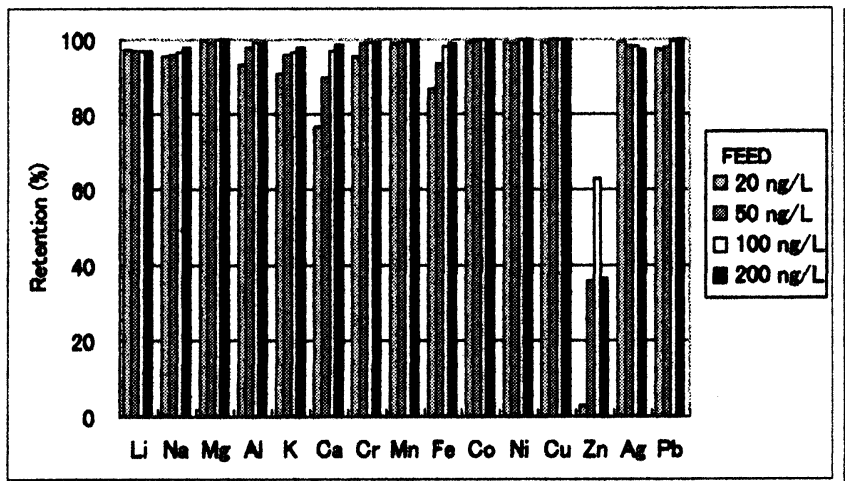

Fig. 2 Retention of the ion exchange cartridge

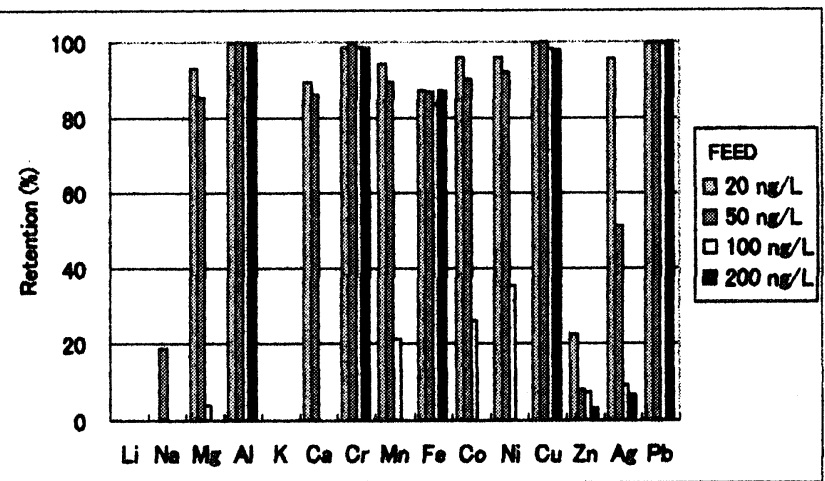

Fig. 3 Retention of the UHMWPE cartridge

metals have around 100 times smaller $\mathrm{K}$ value (equilibrium constant) compared to alkaline earth metals or polyvalent metals. The behavior of the metal retention compared with initial various metal concentration shows no relationship, suggesting that the ion exchange reaction is a first order reaction. In general, this type of reaction between a dilute solution and a solid surface is known as mass transfer control. Because the boundary layer on the surface of the micropores, the fluid is not in convection metal ions need to diffuse through the boundary layer to meet the ion exchange group before the ion exchange reaction can take place.

In the case of $\mathrm{Fe}$ with ion exchange cartridge, the concentration of filtrate was constant on the minimum level of ICP-MS detection. On the other hand, retention of the UHMWPE cartridge was over only $80 \%$. Fe was forming colloidal particles in the experimental conditions. These colloidal particles have large particle size distributions. Smaller particles contain electrical charge, which could be absorbed onto the ion exchange membrane. However the electrical charge density of larger particles were smaller to be absorbed on ion exchange membrane but entrapped on UHMWPE membrane.

$\mathrm{Al}, \mathrm{Cr}, \mathrm{Cu}$ and $\mathrm{Pb}$, the affinity was high enough to be able to be absorbed on the UHMWPE membrane surface and also an ion exchange membrane.

The behavior of $\mathrm{Zn}$ was quite different compared to other metal ions. It is known that zinc carbonate $\left(\mathrm{ZnCO}_{3}\right)$ around neutral $\mathrm{pH}$ has a solubility of few $\mathrm{ng} / \mathrm{dm}^{3}$ as non-electrolyte salt form. [3] It wasn't adsorbed by cation exchange or UHMWPE membrane. $\mathrm{Zn}$ contamination is very hard to be removed with ion exchange membrane as well as ion exchange resin.

The results indicate that the bi-layered structure filter cartridge is very efficient to remove various metal impurities including ionic and colloidal form in UPW. The ionic metal was adsorbed on ion exchange membrane and metal colloidal particles were entrapped by ion exchange filter and microporous membrane such as UHMWPE membrane.

\section{References}

1) Thomas S. Light, Anal.Chem., 1984, 56, 1138-1142.

2) H. Egawa, T. Tanaka, and H. Maeda, J. Appl. Polym. Sci., 30, 3239 (1985)

3) C. F. Baes, Jr. and R. E. Mesmer, “The Hydrolysis of Cations" Robert E. Krieger Publishing Company, Inc. Florida (1986), Chap.13, pp.287-294. 